\section{Validation of the Gel \& Wax Boluses and Comparison of their Dosimetric Performance with Virtual Bolus}

\author{
Verma T. R. ${ }^{1 * \odot}$, Painuly N. K. ${ }^{1}$, Tyagi M. ${ }^{2}$, Johny D. ${ }^{3}$, Gupta \\ R. ${ }^{4}$, Bhatt M. L. B. ${ }^{4}$
}

\begin{abstract}
Background: In general, radiotherapy treatment planning is performed using the virtual bolus. It is necessary to investigate physical bolus in comparison to virtual one.

Objectives: In the present study, first, radiological properties of superflab Gel bolus and Paraffin wax bolus was investigated in terms of their relative electron density. Then, dosimetric performance of both the bolus (i.e. Gel and Parafin wax) was compared with Virtual bolus.
\end{abstract}

Material and Methods: In This experimental study, the radiological property of Wax and Gel boluses was investigated using two methods. In one, the relative electron density of both the Gel and Wax boluses was calculated by measuring their linear attenuation coefficient where in another method relative electron density was calculated by recording their CT No directly from their CT scan. Later CT scan of

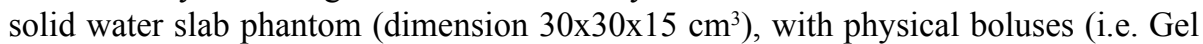
and Wax bolus) of appropriate thicknesses required to deliver a dose of $200 \mathrm{cGy}$ at $\mathrm{D}_{\max }$ using $4 \mathrm{MV}, 6 \mathrm{MV}$ and $15 \mathrm{MV}$ photon beams, was taken. These CT data sets were retrieved to TPS. A plan was done to deliver a dose of $200 \mathrm{cGy}$ at $\mathrm{D}_{\max }$ using Single $4 \mathrm{MV}, 6 \mathrm{MV}$ and $15 \mathrm{MV}$ photon beams. Dose at depths $\mathrm{D}_{\max }, 1 \mathrm{~cm}, 2 \mathrm{~cm}, 3 \mathrm{~cm}, 4 \mathrm{~cm}$ and $5 \mathrm{~cm}$ was recorded. Using this similar method, doses at depths viz $\mathrm{D}_{\max }, 1 \mathrm{~cm}, 2$ $\mathrm{cm}, 3 \mathrm{~cm}, 4 \mathrm{~cm}$ and $5 \mathrm{~cm}$ was recorded for the Gel and Wax boluses. The differences in dose of gel and wax bolus from virtual bolus were recorded for comparison of their dosimetric performance.

Results: The measured (calculated) relative electron density of wax and Gel bolus was found to be $0.958(0.926)$ and $0.923(0.907)$, respectively. Variation in dosimetric performance of Gel and Wax with reference to Virtual bolus was studied. However, on average, Gel bolus was more consistent with virtual bolus.

Conclusion: To avoid any dose difference between, delivered (using physical bolus) and planned (using virtual bolus), the physical boluses should be investigated for their dosimetric performance in comparison to virtual bolus. The results obtained and methodology used in this study can be applied in routine radiotherapy practices.

Citation: Verma T. R, Painuly N. K, Tyagi M, Johny D, Gupta R, Bhatt M. L. B. Validation of the Gel \& Wax Boluses and Comparison of their Dosimetric Performance with Virtual Bolus. J Biomed Phys Eng. 2019;9(6):629-636. https://doi.org/10.31661/jbpe.v0i0.1177.

\section{Keywords}

Paraffin; Tomography, X-Ray Computed; Imaging; Waxes

\section{Introduction}

I $\mathrm{n}$ the radiotherapy technology, water has been the preferred substitute for the generation of data used in dose calculation and helped to deliver accurate radiation dose into every point inside the human
${ }^{1} \mathrm{PhD}$, Department of Radiotherapy, King

George's Medical University, UP, Lucknow, India

${ }^{2} \mathrm{MSc}$, Department of Radiology \& Radiotherapy, UCMS, Delhi, India

${ }^{3} \mathrm{MSc}$, Department of

Radiotherapy, King

George's Medical Univer-

sity, UP, Lucknow, India

${ }^{4} \mathrm{MD}$, Department of

Radiotherapy, King

George's Medical Univer-

sity, UP, Lucknow, India

*Corresponding author:

T. R. Verma

Department of Ra-

diotherapy King George

Medical University, UP,

Lucknow, India

E-mail: Id-

teerth05kashi@gmail.

com

Received: 7 May 2019

Accepted: 15 June 2019 
body [1]. The water phantom used for this purpose is flat, homogeneous, uniform and static in nature. However, dose distribution is affected by the tissue inhomogeneity due to difference in density and atomic number compared to water [2]. Radiation beam incident on an irregular or sloping surface produces tilt in the isodose curves compared to the standard isodose curves obtained from the flat, homogeneous, uniform and static water phantom. However, the surface irregularity may lead dose non-uniformity, which is unacceptable; to rise within the target volume and make OARs such as spinal cord receive excessive irradiation [3, 4]. To deal with this problem, a number of techniques have been devised, including the use of bolus, wedged fields or multiple fields and addition of bolus materials or compensators. Bolus is used to enhance the skin dose in high-energy radiotherapy [5]. One of the requirements of bolus materials is tissue-equivalence in terms of interaction of radiation [6]. To use the bolus as a compensator, with decreased skin sparing effect, it is placed directly on the skin. Ideally, bolus materials should be easy to produce, non- toxic, rigid in the constituent over a periods of use, soft to be located on the skin surface under the beam incident. Moreover, it should provide a homogeneous dose distribution, which covers whole of the target volume. Some of the materials used as bolus are cotton wet, super flab gel, wax bolus etc [7]. Due its frequent use and important role in uniform radiation delivery to target, researchers have always tried to find a bolus material having features such as cost effective and easy to design according to individual patient requirements etc [8]. Furthermore, the performance of bolt material has been found to be affected by the accuracy of the algorithms used in the treatment planning system, too [9].

In this present study, two popular bolus materials namely Gel Bolus and Wax bolus were validated radiologically; then, their dosimetric performance was compared with Virtual bolus found in the treatment planning system.

\section{Material and Methods}

\section{Superflab Gel Bolus}

In this experimental study, superflab gel bolus is available in different thicknesses, which provide maximum dose buildup for relevant photon energies. Since the material is imperceptibly stressed by normal stresses, it does not have to be placed or wrapped in a film to maintain its shape.

At the option of the user, however, superflab may be wrapped in the disposable plastic film for cleanliness and use, or washed with soap and water as needed followed by an application of talcum powder or corn starch. Superflab slabs are elastic and quite flabby. The material conforms to patient's contour while still maintaining good thickness uniformity. Although Super flab exhibits very little cold flows, it is best stored flat, not as folded.

Super flab is an oil gel; thus, it is necessary to provide care. Since the synthetic oils can damage plastic surfaces, particularly vinylcovered furniture.

\section{Wax Bolus Material}

This is a type of pattern wax with pink color. This bolus material is available in the form of sheet of different thicknesses. It is kept in close contact of human skin to enhance the dose deposition towards the anterior side of the incedent beam. Wax bolus is a mixture of some materials namely Paraffin wax (70-80\%), Bee wax (12\%), Carnauba wax (2.5\%), resins $(3 \%)$, and synthetic wax $(2.5 \%)$. It works well over the temperature range of $45-60{ }^{\circ} \mathrm{C}$.

\section{Methodology}

The validation of two boluses (Super flab Gel and Paraffin Wax bolus) was done using the two methods [8-10]. In method No. 1, the HU No. of the bolus materials was recorded directly from their CT scan using the console computer. In method No (2), the CT No. was 
calculated using the formula as following:

$$
\mathrm{HU}=1000\left[\left(\mu_{\mathrm{x}}-\mu_{\mathrm{w}}\right) / \mu_{\mathrm{w}}\right]
$$

Where, $\mu_{\mathrm{x}}$ is the measured linear attenuation coefficient of the bolus material of thickness $\mathrm{x}(\mathrm{cm})$ and $\mu_{\mathrm{w}}$ is the measured linear attenuation coefficient of water. The experimentally measured $\mu_{\mathrm{s}}$ value was then used for calculating the relative electron density of the material using equation

$$
\mu=\left[\log _{\mathrm{e}}\left(\mathrm{N}_{0} / \mathrm{N}_{\mathrm{x}}\right)\right] / \mathrm{x}
$$

To measure the linear attenuation coefficient $\mu$, an experimental setup was used as shown in Figure 1. This setup was comprised of Co60 beam of dimension $2 \times 2 \mathrm{~cm}^{2}$, to incident on the layer of bolus material slab(s) maintained at a distance of $100 \mathrm{~cm}$. To quantify the attenuation capacity of the bolus material under interest, an ionization chamber was put at a distance of $20 \mathrm{~cm}$ (distal to plane over which bolus slabs were placed) along the central axis of the beam. Firstly, the response of the ionization chamber was recorded without any bolus material and this reading of the electrometer was given name $\mathrm{N}_{0}$. After that, same amount of exposure was done with bolus materials slab placed at a distance of $100 \mathrm{~cm}$ from source as shown in Figure 1. The time response of the electrometer was called $\mathrm{N}_{\mathrm{x}}$, where $\mathrm{x}=1 \mathrm{~cm}$ i.e. thickness of slab. Subsequently, meter reading was recorded for different slab thicknesses with increase by one in slab each time. Different values of $\mu$ were measured for various thicknesses; next, the average of the $\mu$ was obtained. The value of $\mu_{\text {water }}$ was also required, as evident from equation (1), to determine CT No. of the bolus materials of interest.

The experimentally measured attenuation coefficient of water i.e. $\mu_{\text {water }}=0.065 \mathrm{~cm}^{-1}$ was used for calculating the relative electron density of the material (bolus) under investigation. This was repeated for both of the bolus materials i.e. Gel bolus and wax bolus.

Moreover, performance of the bolus materials was assessed in clinical setup as well. In which, bolus material in the form of sheet was placed at the surface of water equivalent slab $(\mathrm{SSD}=100 \mathrm{~cm})$ homogeneous phantom of dimension $30 \times 30 \times 15 \mathrm{~cm}^{3}$ as shown in

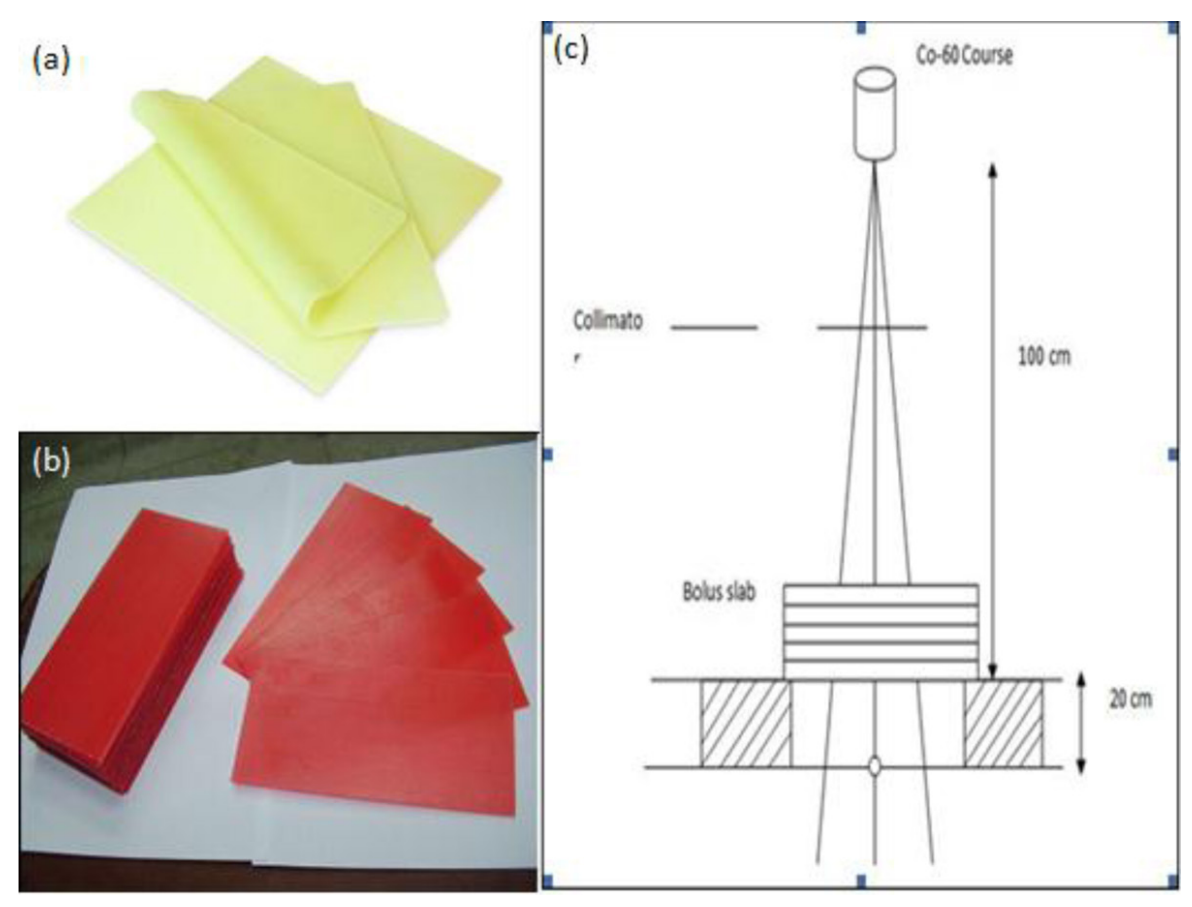

Figure 1: Superflab Gel Bolus (a) Parafin wax Bolus (b) and Experimental setup For the measurement of linear attenuation co-efficient (c) 
Figure 2.

\section{Dosimetric Comparison of Virtual Bolus and Physical Bolus i.e. Wax bolus and Gel}

A solid water slab phantom, of dimension $30 \times 30 \times 15 \mathrm{~cm}^{3}$, was scanned using the settings as followed in real patient. Besides this, the same phantom, with the Wax and Gel boluses of thicknesses $1 \mathrm{~cm}, 1.5 \mathrm{~cm}$ and $2.5 \mathrm{~cm}$ maintained on to the surface of the phantom, was scanned. Then these CT data sets were retrieved to Monaco treatment planning system (V 5.10.04, IMPAC Medical Systems, Inc.,
Maryland Heights, MO) where contouring of the phantom with and without bolus layer was performed. Thus, a treatment plan was created to record the dose deposited along the central axis of $5 \times 5 \mathrm{~cm}^{2}$ photon beam (with gantry and collimator angle $0^{\circ}$ ) for both the setups with physical and virtual boluses of appropriate thickness layers corresponding to energies $4 \mathrm{MV}, 6 \mathrm{MV} \& 15 \mathrm{MV}$ found in the LINAC (ELEKTA Synergy). Dose of 200 cGy was prescribed at $\mathrm{D}_{\max }$; then, dose at depths of 1 $\mathrm{cm}, 2 \mathrm{~cm}, 3 \mathrm{~cm}, 4 \mathrm{~cm} \& 5 \mathrm{~cm}$ along the central axis were recorded in both the setups as shown in Figures 2, 3 and 4.

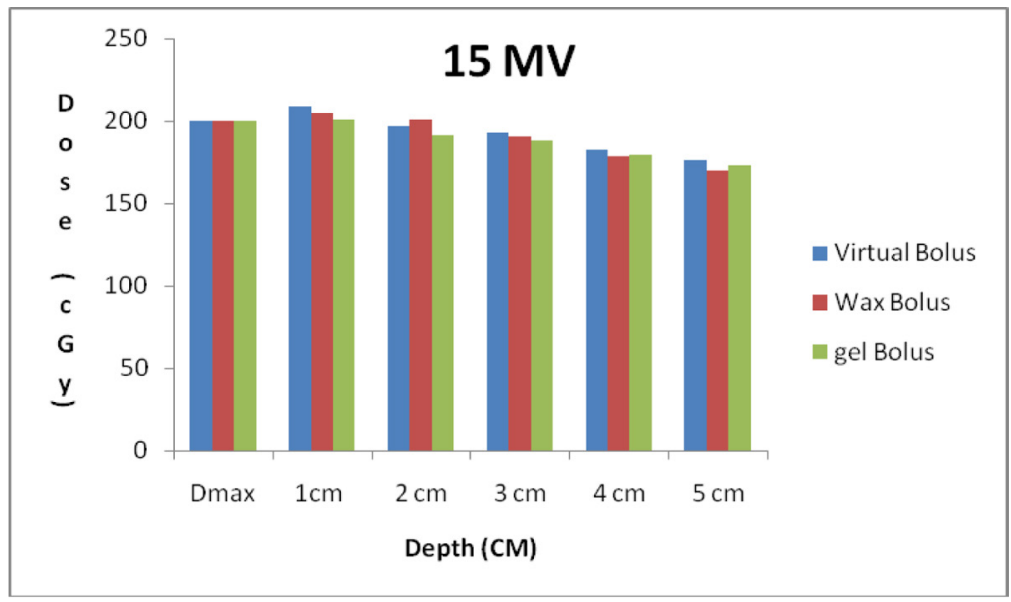

Figure 2: Dose recorded at different depths for $15 \mathrm{MV}$ photon beam

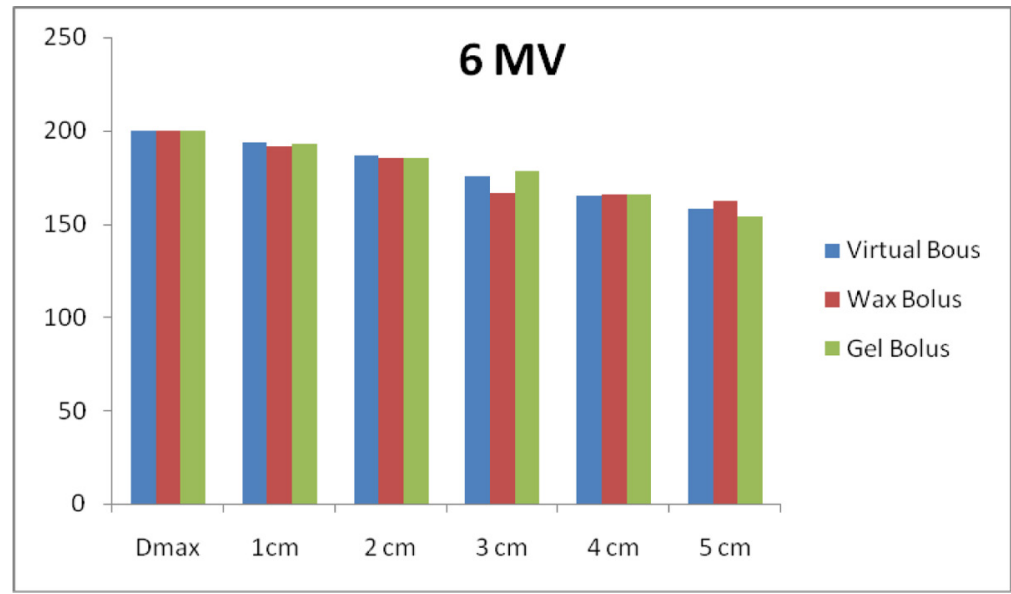

Figure 3: Dose recoreded at different depths for $6 \mathrm{MV}$ photon beam 


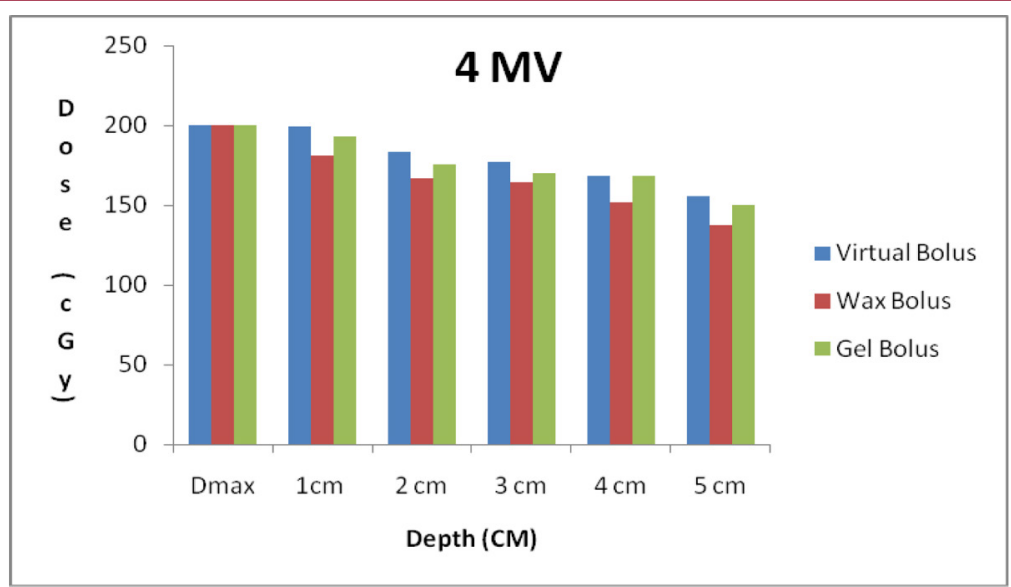

Figure 4: Dose recoreded at different depths for $4 \mathrm{MV}$ photon beam

\section{Results}

\section{Measurement of Relative electron density}

First, validation of both the bolus materials was confirmed. As described under methodology in Eqn No. 2, electrometer response $\mathrm{N}_{0}$ was recorded without putting any wood slabs as shown in Figure 1 (c). The average value of $\mathrm{N}_{0}$ (over the five measurements) was found to be $1.577 \mathrm{nC}\left(\mathrm{N}_{0}=1.577,1.579,1.576\right.$, $1.575 ; \mathrm{N}_{0 \text { mean }}=1.577$ ) with an exposure time of 2 minutes (Table 1). The average value of electrometer reading (with bolus i.e. $\mathrm{N}_{\mathrm{x}}$ ) was recorded to be $0.062 \mathrm{~cm}^{-1}$ and $0.060 \mathrm{~cm}^{-1}$ for paraffin wax and superflab gel boluses, respectively. The values of HU were -42.4 and -76.9 for paraffin wax and superflab gel bous, respectively. The relative electron density of paraffin wax and gel bolus was 0.958 and $0.923 \mathrm{gm} / \mathrm{cm}^{3}$, respectively.

The measured HU No. of the wax and gel boluses were -42.3 and -76.9 whereas the HU values, which directly taken from the $\mathrm{CT}$, was

Table 1: CMeter reading for the measurement of linear attenuation coefficient $(\mu)$

\begin{tabular}{|c|c|c|c|c|c|}
\hline \multicolumn{5}{|c|}{ Meter reading ( $\mathrm{nC}$ ) } & \multirow[t]{2}{*}{$\mu$, mean $\left(\mathrm{cm}^{-1}\right)$} \\
\hline Material Thickness & $0.5 \mathrm{~cm}$ & $1 \mathrm{~cm}$ & $1.5 \mathrm{~cm}$ & $2 \mathrm{~cm}$ & \\
\hline \multirow{5}{*}{ Paraffin Wax } & 1.463 & 1.360 & 1.272 & 1.195 & \multirow{5}{*}{0.062} \\
\hline & 1.467 & 1.364 & 1.270 & 1.192 & \\
\hline & 1.470 & 1.361 & 1.271 & 1.198 & \\
\hline & 1.465 & 1.365 & 1.273 & 1.196 & \\
\hline & 1.463 & 1.362 & 1.273 & 1.195 & \\
\hline \multirow{5}{*}{ Superflab Gel Bolus } & 1.470 & 1.370 & 1.273 & 1.201 & \multirow{5}{*}{0.060} \\
\hline & 1.480 & 1.373 & 1.275 & 1.209 & \\
\hline & 1.472 & 1.365 & 1.270 & 1.208 & \\
\hline & 1.475 & 1.368 & 1.276 & 1.210 & \\
\hline & 1.473 & 1.374 & 1.272 & 1.204 & \\
\hline
\end{tabular}


$-74 \&-93$, respectively. Then after Relative electron density of both the bolus materials was calculated using the equation (1) \& (2) as shown in Table 2.

In addition to radiological properties of both the bolus materials, their performance in the routine clinical practices in terms of dosimetric parameters was assessed. For this, dose (cGy) along the central axis was recorded and compared with the dose recorded for virtual

Table 2: Summary of the radiological parameters of wax and gel boluses

\begin{tabular}{cccccc} 
Bolus Materials & $\begin{array}{c}\text { Mean HU } \\
\text { recorded } \\
\text { from CT scan } \\
{[\mathrm{HU}(\mathrm{CT})]}\end{array}$ & $\begin{array}{c}\text { Relative } \\
\text { electron den- } \\
\text { sity derived } \\
\text { from HU(CT) }\end{array}$ & $\begin{array}{c}\text { Measured } \\
\boldsymbol{\mu}\left(\mathbf{c m}^{-1}\right) \text { in } \\
\text { Telecobalt } \\
\text { beam }\end{array}$ & $\begin{array}{c}\text { Mean HU } \\
\text { measured in } \\
\text { Telecobalt } \\
\text { beam [HU(M)] }\end{array}$ & $\begin{array}{c}\text { Relative } \\
\text { electron den- } \\
\text { sity derived } \\
\text { from HU(M) }\end{array}$ \\
\hline Paraffin wax & -74 & 0.926 & 0.062 & -42.3 & 0.958 \\
\hline Superflab Gel & -93 & 0.907 & 0.060 & -76.9 & 0.923
\end{tabular}

bolus. Dose values were recorded starting of the phantom (i.e. top surface of the phantom) at an interval of $1 \mathrm{~cm}$ up to depth of $5 \mathrm{~cm}$. Dose at depth of $\mathrm{D}_{\max }$ was only $200 \mathrm{cGy}$ in all the mediums, including Wax, super flab gel and Virtual bolus. As for as dosimetric performance of bolus with the change in energy of incident beam, the highest dose difference between the physical and virtual bolus, was obtained for $4 \mathrm{MV}$ photon beam as shown in Figure 4. The highest difference was recorded for the virtual and wax bolus combination. There was not any fixed trend of variation between energy and dose difference. However, on average, the deviation decreased with an increase in energy over the range of $4 \mathrm{MV}$ to $15 \mathrm{MV}$.

Based on the Table 2, paraffin wax has electron density close to water and Superflab gel has lower electron density compared to paraffin wax. As obvious from Table 2, the values of HU No. recorded directly from their CT image, were slightly higher compared to the measured values. In addition, the standard HU No. of the water used in this work was -1000 .

\section{Discussion}

In general, in routine radiotherapy practices, physical bolus (Wax and Gel) has been used as compensator to prevent from the skin sparing effect or shift the dose deposition events (happening inside the medium) towards or onto the skin surface by putting the appropriate thickness of bolus material layer over the desired area of the skin surface.

In the past, the thickness of bolus materials was used to decide based on the quality of the beam employed only. However, in the modern radiotherapy techniques such as 3DCRT, IMRT and other highly conformal radiotherapy techniques, the thickness of the bolus is decided with the help of TPS.

The virtual bolus present in TPS has default relative electron density 1.0. In treatment planning of modern radiotherapy, first, desired dose coverage and dose distribution are finalized using the virtual bolus present in TPS, provided patient was scanned without putting the appropriate bolus. Therefore, it becomes necessary to investigate and validate the dosimetric performance of this virtual bolus.

Alternatively, any difference in dosimetric properties of the physical bolus and virtual bolus may result in deviation of the delivered dose from the planned dose.

In this study, physical bolus was investi- 
gated in terms of CT No. and relative electron density. Humphries et al. compared the dosimetric performance of super stuff and paraffin wax bolus based on the measured dose at various points inside the Rando phantom using the TLDs [7]. This measurement aimed to validate the equivalence of paraffin wax and super stuff since fabrication of paraffin wax bolus is relatively found to be difficult and time-consuming. Although, paraffin wax bolus posses almost the same dosimetric properties as superflab Gel, it has not been used very commonly because of its rigid structure that may result in some air gaps. In this study, performance of the bolus materials was assessed by recording the dose at 6 points along the central axis using three boluses such as superflab gel bolus, paraffin wax bolus and virtual bolus for photon beams of energy 4, 6 \&15 MV. In routine radiotherapy practice, as we decide the radiotherapy planning parameters using the TPS data, dose calculated with virtual bolus is taken as reference in calculation of relative performance of Wax and Gel bolus. As shown in Figures 2, 3, and 4, there was not any fixed trend of variation in dosimetric parameter of bolus with energy of the photon beams used; in addition, wax and gel bolus have been suitable for higher energy photon beams and low energy, respectively.

In general, as evident from Table 3, wax bolus is performing poorly compared to Gel bolus. This table shows the sum of the dose differences between virtual - wax and virtual Gel bolus, for the different photon beams and depth dose points, of 92.9 cGy and 55 cGy, respectively. The highest difference in case of Wax - bolus was contributed by $4 \mathrm{MV}$ photon beam. However, wax bolus has some exclusive uses such as irradiation of penis prohibiting skin sparing effect, and irradiation of structure(s) maintaining its state against gravity.

In general, a virtual bolus is assigned a default electron density of 1 . Also in the current study, the density assigned to the virtual bolus was 1 and this was done to reproduce the actual patient radiotherapy scenario in which radiotherapy planning for the patient in the form of CT scan data is performed using the virtual bolus (default density 1) to achieve the desired dose coverage and dose distribution. Malaescu et al. did a study to investigate the possibility of using the different commercial materials to utilize as bolus material for $6 \mathrm{MeV}$ and $9 \mathrm{MeV}$ electron beam radiotherapy [8].

In this study, bolus materials were investigated using two methods. Other researchers have also used the same methods for validation of the material as lung equivalence [9-11]. In accordance with the Table 2, wax bolus has relative electron density closure to that of water (density of virtual bolus) compared to Gel bolus, though it produced higher dose differences.

Therefore, bolus should be checked before

Table 3: Sum of the differences of dose of same point between virtual bolus and physical bolus

\begin{tabular}{ccccccc} 
& \multicolumn{2}{c}{$\mathbf{6 ~ M V}$} & \multicolumn{2}{c}{$\mathbf{4 ~ M V}$} & \multicolumn{2}{c}{$\mathbf{1 5} \mathbf{~ M V}$} \\
\cline { 2 - 7 } & Wax & Gel & Wax & Gel & Wax & Gel \\
\cline { 2 - 7 } Difference between & 4.2 & 8 & 18.5 & 6.2 & 2.1 & 0.9 \\
\cline { 2 - 7 } $\begin{array}{c}\text { Physical bolus(s) } \\
\text { and Virtual bolus }\end{array}$ & -4.3 & 5.8 & 16.6 & 8.2 & 1.4 & 1.3 \\
\cline { 2 - 7 } & 2.9 & 5.3 & 13.2 & 7.5 & 9.2 & -3.4 \\
\cline { 2 - 7 } & 3.8 & 3.4 & 16.9 & 0.4 & -0.6 & -0.7 \\
\hline Sum of differences & 6.9 & 3 & 17.8 & 5 & -3.8 & 4.1 \\
\hline & 13.6 & 25.5 & 83 & 27.3 & 8.3 & 2.2
\end{tabular}


clinical use to avoid any deviation between delivered dose and planned dose.

\section{Conclusion}

Both Gel and wax bolus were validated radiologically and dosimetrically. Wax bolus has relative electron density close to that of water compared to Gel bolus; however, dosimetric gel bolus should be preferred. The method and results obtained in this study can be implemented in routine radiotherapy practices to avoid any deviation in dose delivered compared to the intended. Furthermore, before using in patient treatment, the bolus materials should be checked for their performance in comparison to virtual bolus. In conclusion, each new material used as bolus must be investigated for its dosimetric performance(s).

\section{Conflict of Interest}

\section{None}

\section{References}

1. El Shahat K, El Saeid A, Attalla E, Yassin A. Comparative Study between Measurement Data and Treatment Planning System (TPS) in Small Fields for High Energy Photon Beams. ISRN Oncol. 2014;2014:901436. doi: 10.1155/2014/901436. PubMed PMID: 24944831; PubMed PMCID: PMCPMC4040206.

2. Catli S. High-density dental implants and radiotherapy planning: evaluation of effects on dose distribution using pencil beam convolution algorithm and Monte Carlo method. J Appl Clin Med Phys. 2015;16:46-52. doi: 10.1120/jacmp. v16i5.5612. PubMed PMID: 26699323; PubMed PMCID: PMCPMC5690165.

3. Kuwahata N, Fujita H, Yamanishi H, Okazaki E, Fukuda H. Dosimetric Comparison of Irregular Surface Compensator and Field-in-Field for Whole Breast Radiotherapy. J Med Phys. 2018;43:79-84. doi: 10.4103/jmp.JMP_73_17. PubMed PMID:
29962684; PubMed PMCID: PMCPMC6020626.

4. Fujita $\mathrm{H}$, Kuwahata N, Hattori $\mathrm{H}$, Kinoshita $\mathrm{H}$, Fukuda $\mathrm{H}$. Improvement of dose homogeneity with irregular surface compensator in whole brain radiotherapy. J Radiother Pract. 2016;15:269-75. doi: $10.1017 / \mathrm{s} 1460396916000212$.

5. Banaee N, Nedaie HA, Nosrati H, Nabavi M, Naderi M. Dose measurement of different bolus materials on surface dose. J Radioprot Res. 2013;1:10-3. doi: 10.12966/jrr.08.02.2013.

6. Tyran M, Tallet A, Resbeut M, Ferre M, Favrel V, Fau $P$, et al. Safety and benefit of using a virtual bolus during treatment planning for breast cancer treated with arc therapy. J Appl Clin Med Phys. 2018;19:463-72. doi: 10.1002/acm2.12398. PubMed PMID: 29959819; PubMed PMCID: PMCPMC6123145.

7. Humphries SM, Boyd K, Cornish P, Newman FD. Comparison of Super Stuff and paraffin wax bolus in radiation therapy of irregular surfaces. Med Dosim. 1996;21:155-7. PubMed PMID: 8899680.

8. Malaescu I, Marin CN, Spunei M. Comparative Study on the Surface Dose of Some Bolus Materials. Int J Med Phys Clin Eng Radiat Oncol. 2015;4:34852. doi: 10.4236/ijmpcero.2015.44041.

9. Verma TR, Painuly NK, Mishra SP, Singh N, Bhatt MLB, Jamal N, et al. Evaluation of dose calculation accuracy of various algorithms in lung equivalent inhomogeneity: Comparison of calculated data with Gafchromic film measured results. J Cancer Res Ther. 2017;13:1007-14. doi: 10.4103/09731482.168992. PubMed PMID: 29237967.

10. Kumar A, Sharma SD, Arya AK, Gupta S, Shrotriya $D$. Effect of low-density heterogeneities in telecobalt therapy and validation of dose calculation algorithm of a treatment planning system. $J$ Med Phys. 2011;36:198-204. doi: 10.4103/09716203.89967. PubMed PMID: 22228928; PubMed PMCID: PMCPMC3249730.

11. Brown S, Bailey DL, Willowson K, Baldock C. Investigation of the relationship between linear attenuation coefficients and CT Hounsfield units using radionuclides for SPECT. Appl Radiat Isot. 2008;66:1206-12. doi: 10.1016/j.apradiso.2008.01.002. PubMed PMID: 18662614. 